\title{
Loss of expression of SDHA predicts SDHA mutations in gastrointestinal stromal tumors
}

\author{
Andrew J Wagner ${ }^{1}$, Stephen P Remillard ${ }^{1}$, Yi-Xiang Zhang ${ }^{1}$, Leona A Doyle ${ }^{2}$, \\ Suzanne George ${ }^{1}$ and Jason L Hornick ${ }^{2}$ \\ ${ }^{1}$ Dana-Farber Cancer Institute, Center for Sarcoma and Bone Oncology, Harvard Medical School, \\ Boston, MA, USA and ${ }^{2}$ Department of Pathology, Brigham and Women's Hospital, \\ Harvard Medical School, Boston, MA, USA
}

\begin{abstract}
Gastrointestinal stromal tumors (GISTs) are usually driven by mutations in KIT or PDGFRA, although $15 \%$ of GISTs in adults and $>90 \%$ in children lack such mutations. The majority of gastric KIT/PDGFRA wild-type GISTs show distinctive morphological and clinical features and loss of expression of succinate dehydrogenase (SDH) B. Only a small subset of SDHB-deficient GISTs carries loss-of-function mutations in SDHB, SDHC, or SDHD. Because of the complexity of its locus (15 exons) and the presence of three pseudogenes, SDHA is rarely analyzed. Recently, mutations in SDHA were shown to lead to loss of expression of SDHA in a small group of paragangliomas. We sought to determine whether immunohistochemistry for SDHA could identify GISTs with SDHA mutations. Tumors $(n=33)$ with pathological features of SDH-deficient GIST were analyzed for expression of SDHA and SDHB by immunohistochemistry, and SDHA exons were sequenced from tumors lacking SDHA expression. Exons harboring somatic mutations were examined in DNA from corresponding normal tissue. All 33 tumors showed loss of SDHB expression. A total of 9 out of $33(27 \%)$ tumors also lacked expression of SDHA. SDHA-deficient GISTs affected five men and four women (median age 38 years). SDHA expression was intact in the 24 remaining tumors, including those with known $\operatorname{SDHB}(n=3)$ or $\operatorname{SDHC}(n=2)$ mutations. Nonsense $(n=8)$ or missense $(n=1)$ mutations in SDHA were identified in all SDHA-deficient tumors. Heterozygous mutations were also found in DNA from normal tissues from six patients with available material. Somatic loss of the second allele has been found in seven tumors, five by loss of heterozygosity, one by a 13-bp deletion, and one by a missense mutation. Loss of SDHA expression in GIST reliably predicts the presence of SDHA mutations, which represent a relatively common cause of SDH-deficient GIST in adults. Immunohistochemistry for SDHA can be used to select patients for SDHA-specific genetic testing.
\end{abstract}

Modern Pathology (2013) 26, 289-294; doi:10.1038/modpathol.2012.153; published online 7 September 2012

Keywords: gastrointestinal stromal tumor; succinate dehydrogenase; SDHA; SDHB; soft tissue sarcoma; immunohistochemistry.

The majority of gastrointestinal stromal tumors (GISTs) contain activating mutations in the KIT or PDGFRA receptor tyrosine kinase genes, ${ }^{1,2}$ which not only drive uncontrolled cellular proliferation and survival but also are the basis for effective targeted therapies with the small molecule kinase inhibitors imatinib and sunitinib. ${ }^{3,4}$ Approximately $15 \%$ of GISTs in adults, and $>90 \%$ of GISTs in children, do not contain identifiable mutations in

Correspondence: Dr JL Hornick, MD, PhD, Department of Pathology, Brigham and Women's Hospital, Harvard Medical School, 75 Francis Street, Boston, MA 02115, USA.

E-mail: jhornick@partners.org

This work was presented in part at the Annual Meeting of the American Society of Clinical Oncology, Chicago, IL, 1-5 June 2012.

Received 6 June 2012; revised 25 July 2012; accepted 26 July 2012; published online 7 September 2012
KIT or PDGFRA and were previously lumped into one group referred to as 'wild-type GIST'. ${ }^{5-7}$ More recently, these tumors have been subcategorized into genetically defined subgroups, including tumors with activating mutations in $B R A F^{8-10}$ loss-offunction mutations in $N F 1$, or loss-of-function mutations in components of the inner mitochondrial membrane Krebs cycle enzyme complex succinate dehydrogenase (SDH). ${ }^{11,12}$ This latter group of tumors has been designated 'SDH-deficient GIST', 13,14 The observed distinctions in molecular genotype have important implications for the biological properties of the tumor, as well as sensitivity to targeted therapies. ${ }^{13,15}$

The SDH complex is comprised of or modified by proteins encoded by $S D H A, S D H B, S D H C$, $S D H D$, and $S D H A F 2 .{ }^{16}$ Germline mutations in these genes have been identified in patients with 
paraganglioma, ${ }^{17-22}$ renal cell carcinoma, ${ }^{23,24}$ and GIST, ${ }^{11,12}$ as well as syndromes of multiple tumor types. $^{11,13}$ In GIST, alterations in SDH have most commonly been reported in $S D H B$, but also have been found in $S D H C, S D H D$, and, recently, $S D H A .{ }^{11,12,25,26}$ Germline mutations in $S D H B$ and $S D H C$ have been identified in SDH-deficient GIST but appear to account for $<15 \%$ of cases. ${ }^{12,14}$ However, mutational analysis of SDHA is seldom performed because of the complex structure of the gene with 15 exons and because of the presence of 3 pseudogenes that make sequencing the proper gene challenging. ${ }^{25}$ Thus, the prevalence of SDHA mutations and consequently the total frequency of any type of SDH subunit gene mutation in GIST are likely underestimated.

SDH-deficient GISTs are characterized by distinctive morphological and clinical features. These KITexpressing tumors arise in the stomach, are comprised of epithelioid or mixed epithelioid and spindled cells, and show a multinodular and infiltrative appearance. $^{14,27}$ Multifocal disease and lymph node metastases are also common, whereas these features are extraordinarily rare in conventional KIT-mutant GISTs. ${ }^{13-15}$ Furthermore, loss of expression of SDHB by immunohistochemistry is a consistent feature of SDH-deficient GIST, whereas SDHB expression is intact in KIT-mutant GISTs. ${ }^{14,27-29}$

A similar genotype/immunophenotype correlation has also been noted in paragangliomas, with mutations in $S D H B, S D H C$, or $S D H D$, leading to loss of SDHB expression. ${ }^{13,30}$ Recently, loss of expression of both SDHA and SDHB by immunohistochemistry in paragangliomas was shown to correlate specifically with mutations in the SDHA gene. ${ }^{31}$ In this study, we sought to determine whether immunohistochemistry for SDHA in SDHdeficient GISTs could similarly predict loss-offunction mutations in $S D H A$.

\section{Materials and methods}

Archival tumor samples were selected for this study on the basis of morphological features consistent with SDH-deficient GIST (gastric origin, epithelioid or mixed morphology, and multinodular/plexiform architecture), according to Institutional Review Board-approved protocols. All patients in this study were diagnosed at $\geq 18$ years of age, because our pathology department and sarcoma oncology group serve an adult patient population.

Immunohistochemistry was performed on $4-\mu \mathrm{m}$ thick formalin-fixed paraffin-embedded whole-tissue sections following pressure cooker antigen retrieval (0.001 M citrate buffer; pH 6.0), using a mouse antiSDHA monoclonal antibody (1:750 dilution; $40 \mathrm{~min}$ incubation; clone 2E3GC12FB2AE2; Abcam, Cambridge, MA) and a mouse anti-SDHB monoclonal antibody (1:100 dilution; $40 \mathrm{~min}$ incubation; clone 21A11AE7; Abcam). The Envision Plus detection system (Dako, Carpinteria, CA) was used as a secondary antibody. Expression was scored as 'intact' when any granular cytoplasmic staining was observed in tumor cells or 'deficient' when there was a complete absence of granular cytoplasmic staining in tumor cells with positive internal controls. Nonneoplastic cells, such as endothelium, smooth muscle, and epithelium, served as internal positive controls.

In cases of SDHA-deficient GIST, genomic DNA was isolated from tumor and corresponding normal tissue (when available) using a QIAamp DNA FFPE Tissue Kit (Cat. No. 56404, Qiagen, Valencia, CA, USA), according to the manufacturer's recommended protocol. Tumor DNA was amplified using intronic primers flanking each of the 15 exons of $S D H A$, designed to avoid known single-nucleotide polymorphisms and amplification of pseudogene sequences (see Supplementary Information for primer details). The resulting amplicons were bidirectionally sequenced by the Sanger method and compared with genomic repository data. Exons with identified somatic mutations were also analyzed in DNA from corresponding normal tissue (when available) to determine germline status.

\section{Results}

A total of 33 cases of GIST were analyzed. The clinical and pathological features of 16 of these cases were previously described. ${ }^{27,32}$ As expected based on selection criteria, all 33 cases were deficient for expression of SDHB. Of these, 9 cases $(27 \%)$ were also deficient for expression of SDHA in tumor cells, whereas expression was maintained in normal epithelial, endothelial, and inflammatory cells in the tissues. Of the patients with SDHAdeficient GISTs, five were male and four were female, with a median age of 38 years (Table 1). None of these patients had a family history of GIST or paraganglioma. SDHA expression was intact in the remaining 24 tumors, including 5 with known mutations in $\operatorname{SDHB}(n=3)$ or $\operatorname{SDHC}(n=2)$ (Figure 1).

Genomic DNA was isolated from the 9 cases of SDHA-deficient GIST, as well as from corresponding normal tissues for which there was adequate material available. PCR amplification and Sanger sequencing of the $S D H A$-coding regions revealed deleterious mutations in all 9 tumors, caused by a single base pair substitution $(n=8)$ or a single base pair deletion $(n=1)$ (Figure 2). Heterozygous mutations were also found in DNA from normal tissue from all six patients with available material. Somatic loss of the second allele was identified in seven out of nine tumors, by loss of heterozygosity in five cases, a 13-bp deletion in one case, and a second somatic mutation in one case (see Figure 2 and Table 2). 
Table 1 Clinical and pathological features of SDHA-deficient gastrointestinal stromal tumors

\begin{tabular}{lclcllll}
\hline Patient & Age at diagnosis & Gender & Tumor size $(\mathrm{cm})$ & Multifocal & Cytology & Vascular invasion & Lymph node metastases \\
\hline 1 & 31 & $\mathrm{M}$ & 4.6 & No & Mixed & Yes & No \\
2 & 38 & $\mathrm{~F}$ & 6.8 & No & Epithelioid & Yes & Yes \\
3 & 39 & $\mathrm{~F}$ & 2 & No & Mixed & No & No \\
4 & 22 & $\mathrm{M}$ & 7.2 (largest) & Yes & Mixed & No & No \\
5 & 53 & $\mathrm{M}$ & 8.5 & No & Epithelioid & No & Yes \\
6 & 41 & $\mathrm{M}$ & 12.5 & No & Epithelioid & No & No \\
7 & 53 & $\mathrm{~F}$ & 3 & No & Mixed & No & No \\
8 & 35 & $\mathrm{~F}$ & 8.5 & No & Epithelioid & Yes & No \\
9 & 19 & M & 12.5 & No & Mixed & Yes & \\
\hline
\end{tabular}
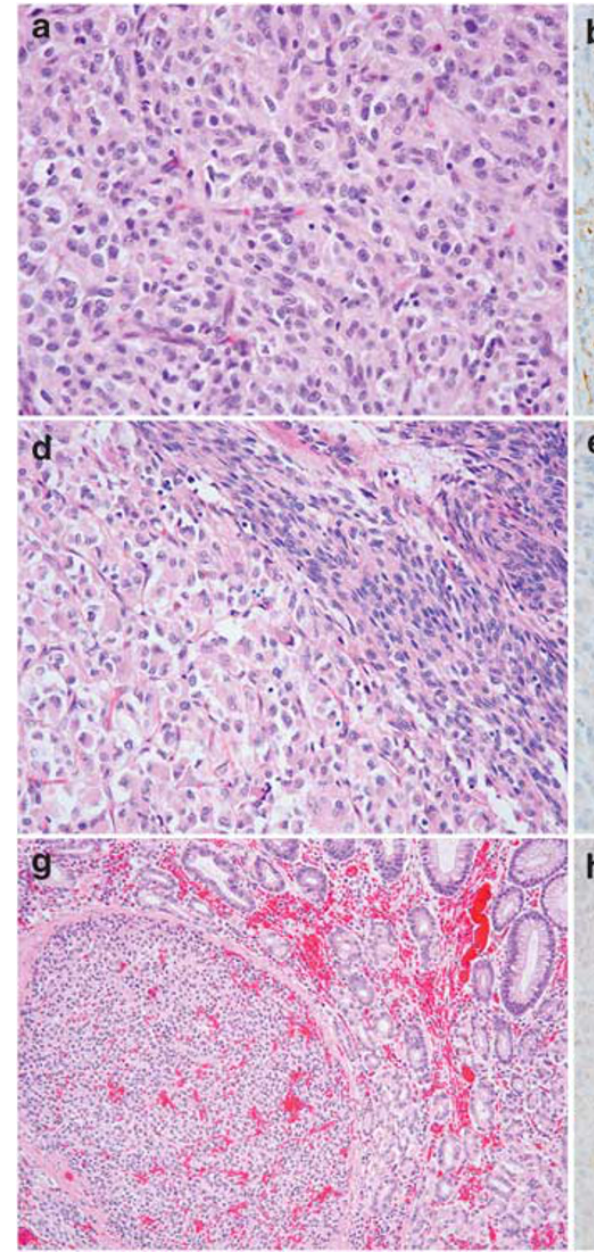

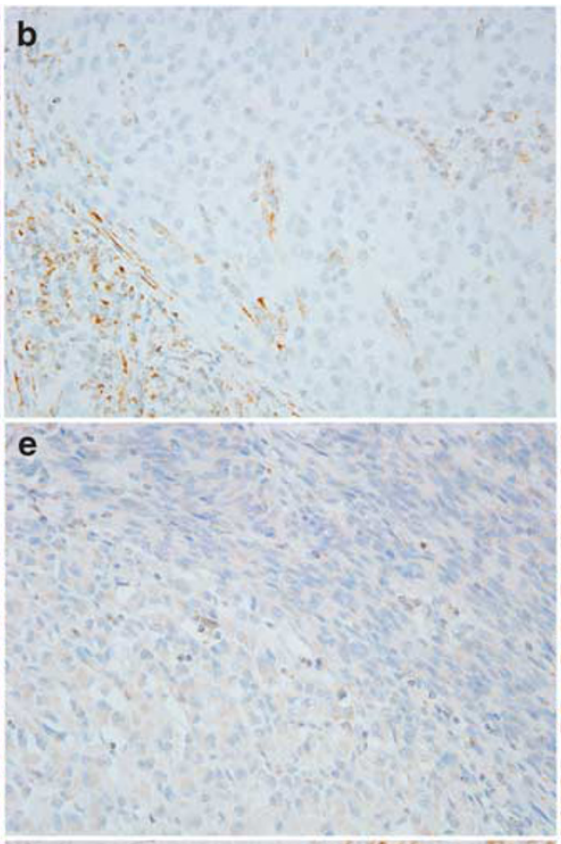

h
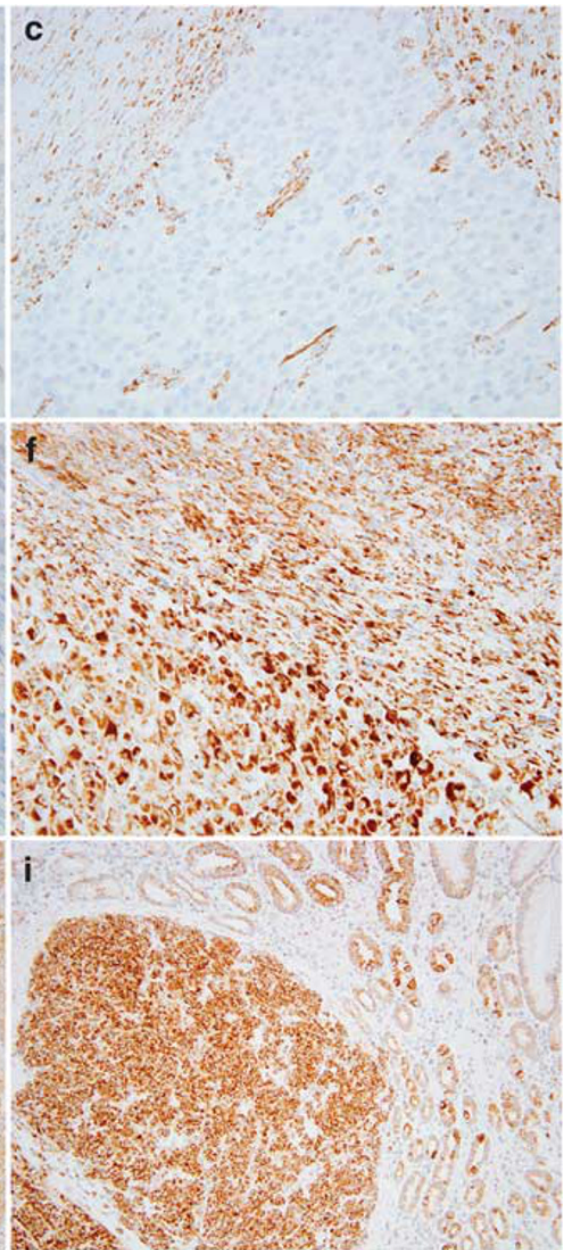

Figure 1 SDH-deficient GISTs of the stomach. (a-c) Epithelioid GIST (a, H\&E) showing loss of expression of both SDHB (b) and SDHA (c) by immunohistochemistry. Note the intact granular cytoplasmic staining in endothelial cells in intratumoral blood vessels, adjacent muscularis propria, and inflammatory cells. This tumor was found to harbor an SDHA mutation. (d-f) SDHB-mutant GIST with mixed epithelioid and spindle cell morphology (d, H\&E) showing loss of expression of SDHB (e) but intact staining for SDHA (f). (g-i) SDHCmutant epithelioid GIST (g, H\&E) showing loss of expression of SDHB (h) but intact staining for SDHA (i). Note the strong cytoplasmic staining in the epithelial cells in the adjacent mucosa.

\section{Discussion}

Loss of expression of the SDHB subunit and SDH activity are universal features of a subset of GISTs characterized by distinctive clinical and morphological features, as well as an absence of mutations in the KIT and PDGFRA genes. ${ }^{11-13,28,29}$ This group of tumors was formerly referred to as 'type 2' or 'pediatric-type' GIST because of their distinctive pathological features, wild-type KIT and PDGFRA status, and prevalence among GISTs arising in patients $<18$ years of age ${ }^{29,32}$ Because this type of GIST also arises in adult patients (overall accounting for the majority of such tumors), the term 


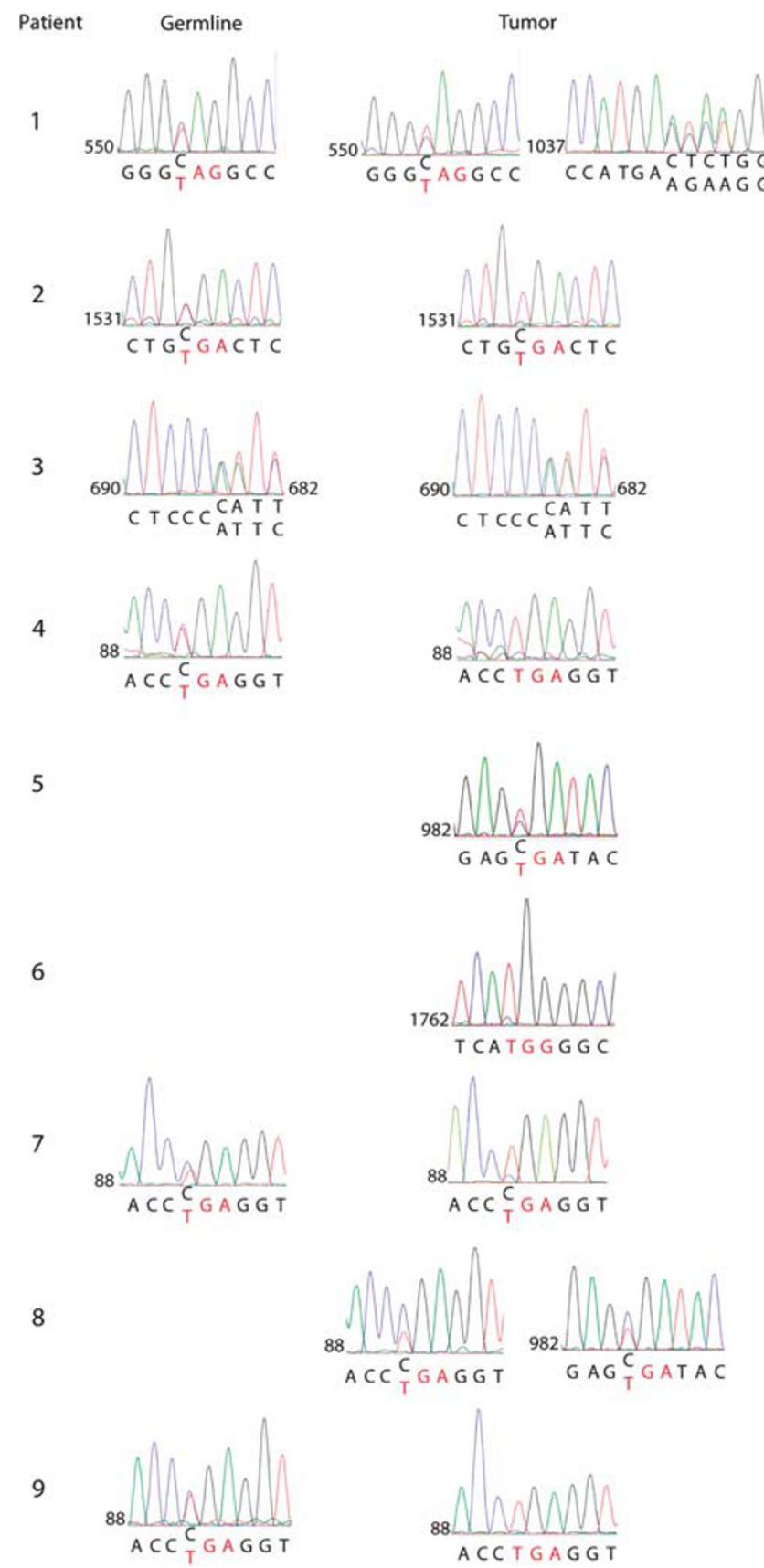

Figure 2 Sequencing chromatograms of selected $S D H A$ exons from normal (germline) and tumor tissue. Numbers represent positions of indicated nucleotides. For patient 3, the reverse complement strand is shown for clarity of chromatogram depiction. See Table 2 for mutation details.

'SDH-deficient GIST' is increasingly applied, reflecting the underlying biochemical and, in some cases, genetic lesions. ${ }^{12-14}$

In one report, germline mutations in $S D H B$ or SDHC were identified in $12 \%$ of 34 SDH-deficient tumors, without pathogenic mutations identified in $S D H D .{ }^{12}$ Of note, no mutations in $S D H B, S D H C$, or $S D H D$ were identified in another recent study, although only a subset of exons was sequenced. ${ }^{14}$ Mutations in SDHA have also recently been found in several unselected patients with KIT/PDGFRA wildtype GIST. ${ }^{25,26}$ However, the SDHA gene is not routinely interrogated for mutations both because of the perception that it is infrequently mutated and also because of the technical challenges in sequencing a gene with 15 exons and 3 pseudogenes. ${ }^{12,25}$

Although the diagnosis of SDH-deficient GIST can be made on the basis of morphological features and loss of SDHB expression, ${ }^{14,27}$ characterization of the particular genotype has important implications for screening for additional tumor types as well as for genetic counseling and identification of similarly affected family members. Differing SDH subunit mutations are associated with location-specific paraganglioma (in particular, $S D H D$ and head and neck tumors, and $S D H B$ and thoracoabdominal tumors) and renal cell carcinoma (especially $S D H B$ ), for example, and therefore screening for occult tumors may be influenced by the particular genetic alterations. ${ }^{13,24,33-35}$ Of note, unlike other SDH subunits, there does not appear to be a site predilection for SDHA-mutant paragangliomas, as there is a wide reported anatomic distribution (abdominal, bladder, thoracic, vagal, and carotid body paragangliomas, as well as a pheochromocytoma). ${ }^{31}$ Additionally, identification of the precise mutation in a proband would facilitate screening of family members. However, it is notable that germline mutations in SDHA, unlike mutations in other SDH subunit genes, have not thus far been associated with a familial tumor syndrome. The reported patients with SDHA-mutant paragangliomas have had apparently sporadic tumors, ${ }^{31}$ and none of the patients with SDHA-mutant GISTs in our current study had a family history of either paraganglioma or GIST. Furthermore, no patients with germline SDHA mutations and both GIST and paraganglioma have yet been reported (in contrast to the Carney-Stratakis syndrome with germline mutations in $S D H B, S D H C$, or $S D H D$ ). The same $S D H A$ mutations reported in paragangliomas have also been identified at a low rate in healthy donors; ${ }^{31}$ it therefore seems likely that there is a low penetrance of both GISTs and paragangliomas in patients with germline SDHA mutations. Until additional followup and complete family history are obtained on a larger cohort of patients with $S D H A$-mutant GISTs, the familial implications of identifying a germline SDHA mutation remain somewhat uncertain.

In our study, we have found that expression of SDHA is lost in $27 \%$ of SDH-deficient GISTs, and that, similar to paraganglioma, ${ }^{31}$ loss of SDHA expression in tumors reliably predicts the presence of SDHA mutations in tumor cells and associated germline material. Thus, immunohistochemistry can help focus germline testing on SDHA. Interestingly, the most common mutation in our patient cohort (c.91C > T; p.R31X), found in four tumors, was previously reported in one of four patients with SDHA-mutant GIST ${ }^{26}$ and is also the most frequently reported $S D H A$ mutation in paragangliomas. ${ }^{31}$ Of 
Table 2 Germline and somatic mutations identified in nine patients with SDHA-deficient gastrointestinal stromal tumor

\begin{tabular}{|c|c|c|c|c|c|c|}
\hline \multirow[t]{2}{*}{ Sample } & \multicolumn{3}{|c|}{ Tumor tissue mutation(s) } & \multicolumn{3}{|c|}{ Normal tissue mutation } \\
\hline & SDHA exon & Nucleotide & Amino acid & SDHA ехоп & Nucleotide & Amino acid \\
\hline 1 & $\begin{array}{l}\text { Exon } 5 \\
\text { Exon } 8\end{array}$ & $\begin{array}{l}\text { c. } 553 \mathrm{C}>\mathrm{T} \\
\text { c. } 1043-1055 \mathrm{del}\end{array}$ & $\begin{array}{l}\text { p.Q185X } \\
\text { Frameshift }\end{array}$ & Exon 5 & c. $553 \mathrm{C}>\mathrm{T}$ & p.Q185X \\
\hline 2 & $\begin{array}{l}\text { Exon } 11 \\
\text { Loss of heterozygosity }\end{array}$ & c. $1534 \mathrm{C}>\mathrm{T}$ & p.R512X & Exon 11 & c. $1534 \mathrm{C}>\mathrm{T}$ & p.R512X \\
\hline 3 & $\begin{array}{l}\text { Exon } 8 \\
\text { None detected }\end{array}$ & c.688delG & Frameshift & Exon 8 & c.688delG & Frameshift \\
\hline 4 & $\begin{array}{l}\text { Exon } 2 \\
\text { Loss of heterozygosity }\end{array}$ & c. $91 \mathrm{C}>\mathrm{T}$ & p.R31X & Exon 2 & c. $91 \mathrm{C}>\mathrm{T}$ & p.R31X \\
\hline 5 & $\begin{array}{l}\text { Exon } 8 \\
\text { None detected }\end{array}$ & c. $985 \mathrm{C}>\mathrm{T}$ & p.R329X & Not available & & \\
\hline 6 & $\begin{array}{l}\text { Exon } 13 \\
\text { Loss of heterozygosity }\end{array}$ & c. $1765 \mathrm{C}>\mathrm{T}$ & p.R589W & Not available & & \\
\hline 7 & $\begin{array}{l}\text { Exon } 2 \\
\text { Loss of heterozygosity }\end{array}$ & c. $91 \mathrm{C}>\mathrm{T}$ & p.R31X & Exon 2 & c. $91 \mathrm{C}>\mathrm{T}$ & p.R31X \\
\hline 8 & $\begin{array}{l}\text { Exon } 2 \\
\text { Exon } 8\end{array}$ & $\begin{array}{l}\text { c. } 91 \mathrm{C}>\mathrm{T} \\
\text { c. } 985 \mathrm{C}>\mathrm{T}\end{array}$ & $\begin{array}{l}\text { p.R31X } \\
\text { p.R329X }\end{array}$ & Not available & & \\
\hline 9 & $\begin{array}{l}\text { Exon } 2 \\
\text { Loss of heterozygosity }\end{array}$ & c. $91 \mathrm{C}>\mathrm{T}$ & p.R31X & Exon 2 & c. $91 \mathrm{C}>\mathrm{T}$ & p.R31X \\
\hline
\end{tabular}

note, three patients from our study with confirmed SDHA mutations underwent clinical sequencing of $S D H B, S D H C$, and $S D H D$, all of which were negative. The prevalence of detected $S D H A$ mutations in our cohort suggests that alterations in this gene are likely more common than those in $S D H B, S D H C$, and $S D H D,{ }^{12}$ although this finding needs to be confirmed in a larger series. Although these studies together suggest that only $35-40 \%$ of SDH-deficient GISTs contain mutations in an SDH subunit, undetected mutations, deletions or epigenetic alterations of SDH subunit genes, or possibly mutations in genes encoding other cofactor proteins may account for the remaining cases of SDH deficiency. Further identification of the genetic mechanisms leading to SDHdeficient GIST will facilitate genetic classification of these tumors and will help guide mutation-specific cancer screening and genetic counseling.

\section{Acknowledgements}

We are grateful to Dr Esther Korpershoek (University Medical Center, Rotterdam) for providing SDHA primer sequences, and to the DK Ludwig Fund for Cancer Research for support of the Ludwig Center at Dana-Farber/Harvard.

\section{Disclosure/conflict of interest}

The authors declare no conflict of interest.

\section{References}

1 Marrari A, Wagner AJ, Hornick JL. Predictors of response to targeted therapies for gastrointestinal stromal tumors. Arch Pathol Lab Med 2012;136:483-489.

2 Rubin BP, Heinrich MC, Corless CL. Gastrointestinal stromal tumour. Lancet 2007;369:1731-1741.

3 Demetri GD, van Oosterom AT, Garrett CR, et al. Efficacy and safety of sunitinib in patients with advanced gastrointestinal stromal tumour after failure of imatinib: a randomised controlled trial. Lancet 2006; 368:1329-1338.

4 Demetri GD, von Mehren M, Blanke CD, et al. Efficacy and safety of imatinib mesylate in advanced gastrointestinal stromal tumors. N Engl J Med 2002;347:472-480.

5 Liegl-Atzwanger B, Fletcher JA, Fletcher CD. Gastrointestinal stromal tumors. Virchows Arch 2010;456: 111-127.

6 Prakash S, Sarran L, Socci N, et al. Gastrointestinal stromal tumors in children and young adults: a clinicopathologic, molecular, and genomic study of 15 cases and review of the literature. J Pediatr Hematol Oncol 2005;27:179-187.

7 Agaram NP, Laquaglia MP, Ustun B, et al. Molecular characterization of pediatric gastrointestinal stromal tumors. Clin Cancer Res 2008;14:3204-3215.

8 Agaimy A, Terracciano LM, Dirnhofer S, et al. V600E BRAF mutations are alternative early molecular events in a subset of KIT/PDGFRA wild-type gastrointestinal stromal tumours. J Clin Pathol 2009;62: 613-616.

9 Agaram NP, Wong GC, Guo T, et al. Novel V600E BRAF mutations in imatinib-naive and imatinib-resistant gastrointestinal stromal tumors. Genes Chromosomes Cancer 2008;47:853-859. 
10 Hostein I, Faur N, Primois C, et al. BRAF mutation status in gastrointestinal stromal tumors. Am J Clin Pathol 2010;133:141-148.

11 Pasini B, McWhinney SR, Bei T, et al. Clinical and molecular genetics of patients with the Carney-Stratakis syndrome and germline mutations of the genes coding for the succinate dehydrogenase subunits SDHB, SDHC, and SDHD. Eur J Hum Genet 2008;16: 79-88.

12 Janeway KA, Kim SY, Lodish M, et al. Defects in succinate dehydrogenase in gastrointestinal stromal tumors lacking KIT and PDGFRA mutations. Proc Natl Acad Sci USA 2011;108:314-318.

13 Barletta JA, Hornick JL. Succinate dehydrogenasedeficient tumors: diagnostic advances and clinical implications. Adv Anat Pathol 2012;19:193-203.

14 Miettinen M, Wang ZF, Sarlomo-Rikala M, et al. Succinate dehydrogenase-deficient GISTs: a clinicopathologic, immunohistochemical, and molecular genetic study of 66 gastric GISTs with predilection to young age. Am J Surg Pathol 2011;35:1712-1721.

15 Gill AJ. Succinate dehydrogenase (SDH) and mitochondrial driven neoplasia. Pathology 2012;44: 285-292.

16 Bardella C, Pollard PJ, Tomlinson I. SDH mutations in cancer. Biochim Biophys Acta 2011;1807:1432-1443.

17 Astuti D, Douglas F, Lennard TW, et al. Germline SDHD mutation in familial phaeochromocytoma Lancet 2001;357:1181-1182.

18 Gimm O, Armanios M, Dziema H, et al. Somatic and occult germ-line mutations in SDHD, a mitochondrial complex II gene, in nonfamilial pheochromocytoma. Cancer Res 2000;60:6822-6825.

19 Mannelli M, Castellano M, Schiavi F, et al. Clinically guided genetic screening in a large cohort of italian patients with pheochromocytomas and/or functional or nonfunctional paragangliomas. J Clin Endocrinol Metab 2009;94:1541-1547.

20 Neumann HP, Bausch B, McWhinney SR, et al. Germline mutations in nonsyndromic pheochromocytoma. N Engl J Med 2002;346:1459-1466.

21 Niemann S, Muller U. Mutations in SDHC cause autosomal dominant paraganglioma, type 3. Nat Genet 2000;26:268-270.

22 Taschner PE, Jansen JC, Baysal BE, et al. Nearly all hereditary paragangliomas in the Netherlands are caused by two founder mutations in the SDHD gene. Genes Chromosomes Cancer 2001;31:274-281.

23 Gill AJ, Pachter NS, Chou A, et al. Renal tumors associated with germline SDHB mutation show distinctive morphology. Am J Surg Pathol 2011;35: 1578-1585.
24 Gill AJ, Pachter NS, Clarkson A, et al. Renal tumors and hereditary pheochromocytoma-paraganglioma syndrome type 4. N Engl J Med 2011;364:885-886.

25 Pantaleo MA, Astolfi A, Indio V, et al. SDHA loss-offunction mutations in KIT-PDGFRA wild-type gastrointestinal stromal tumors identified by massively parallel sequencing. J Natl Cancer Inst 2011;103:983-987.

26 Pantaleo MA, Nannini M, Astolfi A, et al. A distinct pediatric-type gastrointestinal stromal tumor in adults: potential role of succinate dehydrogenase subunit A mutations. Am J Surg Pathol 2011;35:1750-1752.

27 Doyle LA, Nelson D, Heinrich MC, et al. Loss of succinate dehydrogenase subunit B (SDHB) expression is limited to a distinctive subset of gastric wild-type gastrointestinal stromal tumours: a comprehensive genotype-phenotype correlation study. Histopathology 2012;doi:10.1111/j.1365-2559.2012.04300.x.

28 Gaal J, Stratakis CA, Carney JA, et al. SDHB immunohistochemistry: a useful tool in the diagnosis of Carney-Stratakis and Carney triad gastrointestinal stromal tumors. Mod Pathol 2011;24:147-151.

29 Gill AJ, Chou A, Vilain R, et al. Immunohistochemistry for SDHB divides gastrointestinal stromal tumors (GISTs) into 2 distinct types. Am J Surg Pathol 2010;34: 636-644.

30 van Nederveen FH, Gaal J, Favier J, et al. An immunohistochemical procedure to detect patients with paraganglioma and phaeochromocytoma with germline SDHB, SDHC, or SDHD gene mutations: a retrospective and prospective analysis. Lancet Oncol 2009;10:764-771.

31 Korpershoek E, Favier J, Gaal J, et al. SDHA immunohistochemistry detects germline SDHA gene mutations in apparently sporadic paragangliomas and pheochromocytomas. J Clin Endocrinol Metab 2011;96:E1472E1476.

32 Rege TA, Wagner AJ, Corless CL, et al. 'Pediatric-type' gastrointestinal stromal tumors in adults: distinctive histology predicts genotype and clinical behavior. Am J Surg Pathol 2011;35:495-504.

33 Amar L, Baudin E, Burnichon N, et al. Succinate dehydrogenase B gene mutations predict survival in patients with malignant pheochromocytomas or paragangliomas. J Clin Endocrinol Metab 2007;92:3822-3828.

34 Baysal BE, Willett-Brozick JE, Lawrence EC, et al. Prevalence of SDHB, SDHC, and SDHD germline mutations in clinic patients with head and neck paragangliomas. J Med Genet 2002;39:178-183.

35 Benn DE, Gimenez-Roqueplo AP, Reilly JR, et al. Clinical presentation and penetrance of pheochromocytoma/paraganglioma syndromes. J Clin Endocrinol Metab 2006;91:827-836.

Supplementary Information accompanies the paper on Modern Pathology website (http://www.nature.com/ modpathol) 